\title{
Análisis energético y ambiental de alumbrado inteligente: Instalación en la Facultad de Derecho de la Universidad de Santiago de Compostela
}

\author{
Fernando BLANCO SILVA \\ Universidad de Santiago de Compostela \\ fernando.blanco.silva@usc.es \\ Gabriel PEREIRO LÓPEZ \\ Grupo de Ecoeficiencia \\ Universidad de Santiago de Compostela \\ pereiro.gabriel@gmail.es \\ Alfonso LÓPEZ DÍAZ \\ Facultad de Ciencias y Artes \\ Universidad Católica de Ávila \\ alfonso.lopez@ucavila.es
}

Recibido: 6 de mayo de 2013

Enviado a evaluar: 15 de mayo de 2013

Aceptado: 3 de octubre de 2013

\section{RESUMEN}

El suministro de energía es un serio problema para los países que carecen de fuentes propias de energía, como es el caso de España. Desde la década de los setenta del pasado siglo el precio de la energía ha subido de forma constante y es necesario que se realicen acciones para minimizar éste. En el presente artículo presentamos un ejemplo de ello, las actuaciones llevadas a cabo en el edificio Facultad de Derecho de la Universidad de Santiago de Compostela (USC) que buscan minimizar el consumo de energía en alumbrado. Las tareas consistieron en una renovación del alumbrado en la Sala de Lectura, así como la instalación de un alumbrado regulado por células fotoeléctricas en pasillos y vestíbulos.

Palabras clave: Ahorro de energía, emisiones de gases de efecto invernadero y alumbrado.

Energy and environmental analysis intelligent lighting: Installation in the Faculty of Law of the University of Santiago de Compostela

\begin{abstract}
The supply of energy is a serious problem for countries that lack their own sources of energy, as is the case in Spain. Since the seventies of last century the price of energy has risen steadily and it is necessary to take actions to minimize power consumption. In this paper you can see an example of this, specifically, the actions carried out in the building of the Faculty of Law, University of Santiago (USC) in order to minimize power consumption. The tasks consisted of a renewal of the lighting in the Reading Room and the installation of lighting controlled by photocells in corridors and lobbies.
\end{abstract}

Key words: Energy saving, emissions of greenhouse gases and lighting. 


\author{
L'analyse de l'énergie d'éclairage intelligent: \\ Installation de la Faculté de droit Université de Saint Jacques de Compostelle
}

\title{
RÉSUMÉ
}

El l'approvisionnement en énergie est un problème grave pour les pays qui n'ont pas de sources d'énergie, comme c'est le cas en Espagne. Depuis les années soixante-dix du siècle dernier, le prix de l'énergie n'a cessé d'augmenter et il est nécessaire de prendre des mesures pour réduire la consommation. Dans cet article, nous présentons un exemple de cela, les actions menées à la Faculté du bâtiment de droit, Université de Saint-Jacques-de-Compostelle (USC) qui visent à réduire la consommation d'énergie dans l'éclairage. Les tâches consistent en une rénovation de l'éclairage public de la salle de lecture, et l'installation d'un éclairage contrôlé par des photocellules electriques à las couloirs et les halls.

Mots-clés: économie d'énergie, les émissions de gaz à effet et l'éclairage.

\section{INTRODUCCIÓN: LA CRISIS ENERGÉTICA DEL SIGLO XXI}

El primer paso a la hora de estudiar una mejora energética es entender el contexto histórico, y para esto debemos remontarnos a las Crisis del Petróleo (1973 y 1979); en estos años los países productores provocaron subidas espectaculares en el precio del hidrocarburo afectando a todas las economías mundiales, especialmente a los países que no disponían de este recurso (Ballenilla, F., 2004). Hasta los años setenta el petróleo no supone un coste importante en los procesos productivos, pero la primera subida de los precios (desde 1,6 \$ hasta 9 \$ en 1973) (Ruíz Bevía, F., 1981) incluye un coste adicional que distorsiona el sector industrial; después de esta subida los países importadores buscaron alternativas a esta dependencia, además de introducir un nuevo coste en el proceso productivo hay un problema estratégico, ya que están indefensos ante nuevas subidas de precios del hidrocarburo y por extensión de cualquier combustible. Después de la segunda Crisis (1979) los países afectados optan por diversas estrategias que les blinden ante nuevas subidas de precios, aunque el margen de actuación es limitado porque en muchos casos no existían alternativas tecnológicas (en particular para el transporte), con escasa implantación (gas natural) o eran tecnologías no maduras (renovables), así para disminuir la dependencia del crudo se fomenta el "mix energético", es decir la diversificación de fuentes; en esta situación se encontraron España, Italia, Argentina, Corea del Sur, Francia o Japón (British Petroleum, 2011; Amestoy Alonso, J., 2000; Bustelo, P., 2008). En la década de los ochenta se opta por el gas natural y la energía nuclear (en particular Francia y Japón), mientras que a partir de la década de los noventa la apuesta se centra en el consumo de fuentes autóctonas, la eficiencia y el ahorro de energía.

Otro hecho importante en el contexto estudiado es la firma del Protocolo de Kyoto para la reducción de las emisiones de Gases de Efecto Invernadero (G.E.I.). En 1997 se firmó en Kyoto (Japón) un acuerdo entre los países más industrializados para detener las emisiones de Gases de Efecto Invernadero, que son el dióxido de carbono y otros cinco gases menos comunes. Estos gases provocan el aumento de la temperatura media del planeta, conocido como Calentamiento Global, siendo el principal 
motivo la combustión de hidrocarburos (Campos Hernández, J. M., 2008; Fernández López, M.A. et alii, 2008): $\mathrm{CnHm}+\mathrm{O}_{2} \rightarrow \mathrm{CO}_{2}+\mathrm{H}_{2} \mathrm{O}+\mathrm{Q}$.

El Protocolo busca reducir las emisiones de los países más industrializados (EEUU, Rusia, Australia, Unión Europea,...) en el período 2008-2012 a los niveles de emisión de $\mathrm{CO}_{2}$ en 1990 y a los de 1995 para el resto de G.E.I. Para evitar las emisiones masivas de $\mathrm{CO}_{2}$ las opciones más recomendables son el uso de fuentes que no producen emisiones (renovables o energía nuclear), el aumento de la importancia del gas natural (su combustión es más limpia y eficiente que el carbón y petróleo, recomendándose la implantación de ciclos combinados en centrales termoeléctricas sustituyendo al gas natural), el ahorro de energía y la mejora de la eficiencia energética.

El tercer aspecto a considerar es el aumento del consumo de energía. Desde los años ochenta España ha aprobado herramientas para reducir el consumo de energía (Delgado-Iribarren, M. 1984), aunque el éxito ha sido escaso. El acceso generalizado de la población a las redes eléctricas, el aumento de bienestar y sectores productivos poco eficientes han provocado un aumento en el consumo durante las últimas décadas. En la Figura 1 se puede ver la evolución del consumo de energía a lo largo de las últimas décadas; en los momentos de expansión económica el consumo es creciente, mientras que en recesión el consumo se frena. Las nuevas herramientas jurídicas proponen la mejora de la eficiencia energética aun considerando creciente el consumo de energía, ya que sería muy difícil mantener la competitividad con otros países si se redujese el consumo de energía (Sánchez Tembleque, L. J., 2011).

Figura 1. Evolución de la energía primaria consumida en España (ktep).

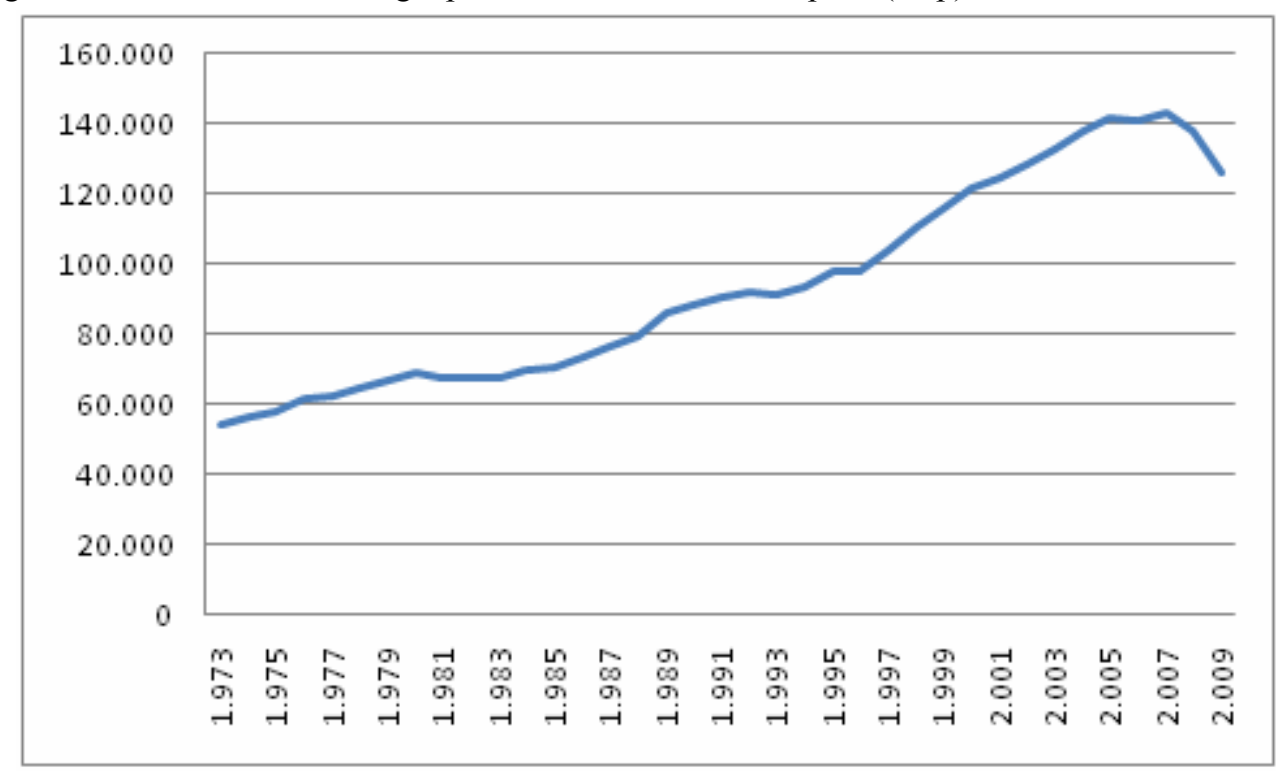

Fuente: Anuario de la Energía 2011 de Foro Nuclear. 
En función de los tres aspectos citados (dependencia del petróleo y recursos propios, emisiones de $\mathrm{CO}_{2}$ y aumento del consumo de energía) se definen las políticas energéticas de cada país, adaptándose a los recursos propios. En el caso de España, las últimas tendencias en cuanto a fuentes de energía son:

- Promover las fuentes de energía renovables, en particular la eólica.

- Aumentar la presencia del gas natural; principalmente por dos vías, en usos térmicos (calderas domésticas e industriales) y en termoeléctricos (sustitución de centrales de vapor de bajo rendimiento por otras de ciclo combinado más eficientes).

- Mantener los consumos totales de petróleo y energía nuclear, aunque el porcentaje de éstos en la energía primaria vaya descendiendo.

- Asegurar un porcentaje mínimo de carbón de origen nacional por motivos estratégicos (aunque su coste sea superior a otras fuentes), como son asegurar un mínimo de empleo en las zonas mineras y mantener la diversificación.

A día de hoy, la estructura energética española es la mostrada en la Figura 2.

Figura 2. Fuentes de energía en el balance nacional de energía primaria (2010)

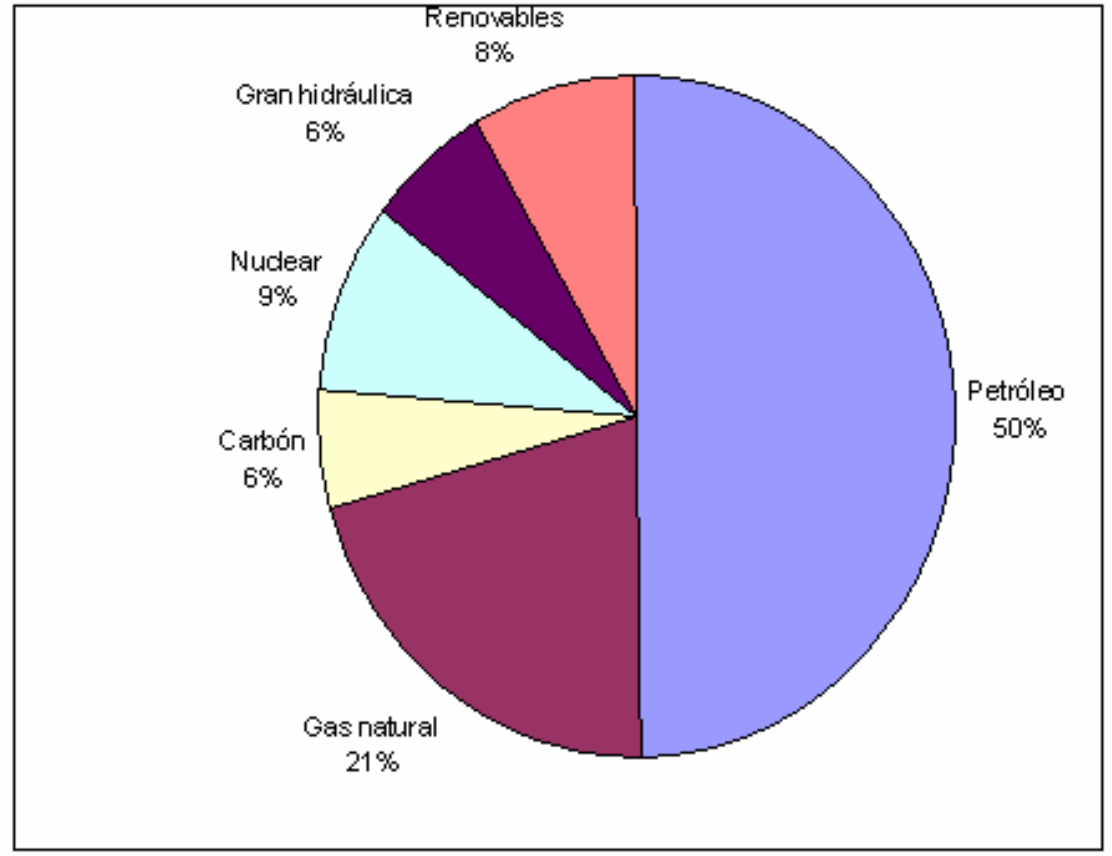

Fuente: Anuario 2011 de Foro Nuclear. 


\section{DESCRIPCIÓN DE LA INSTALACIÓN ELÉCTRICA DE LA FACULTAD DE DERECHO DE SANTIAGO DE COMPOSTELA}

\subsection{DATOS GENERALES DEL EDIFICIO}

La Facultad de Derecho se emplaza en la Avenida Ángel Jorge Echeverri, S/N, situada en el Campus Sur de Santiago de Compostela. Esta ciudad está en el Hemisferio Norte con latitud de $42^{\circ} 52^{\prime} \mathrm{N}$ y longitud de $8^{\circ} 33^{\prime} \mathrm{O}$, en la provincia de La Coruña. Este edificio se construyó en la década de los setenta y actualmente se destina al desarrollo de actividades académicas, principalmente para la docencia de la Licenciatura de Derecho. Otras actividades formativas impartidas en el edificio son la titulación propia de Criminología, Estudios de Tercer Ciclo, Escuela de Práctica Jurídica, Máster en Administración Local, Cursos de Especialización,... así como, las tareas administrativas relacionadas con éstos (Decanato, Negociado de Asuntos Económi$\cos , \ldots)$. En el edificio no existen procesos importantes a nivel de laboratorios o de equipos de alto consumo eléctrico, por lo que el mayor consumo de energía eléctrica se centra en el alumbrado, seguido de estufas, equipos informáticos y, ya de forma puntual, dos instalaciones de aire acondicionado. Durante el curso lectivo el edificio está abierto de lunes a viernes desde las $8 \mathrm{~h}$ a las $22 \mathrm{~h}$ y los sábados por la mañana. El horario de pleno funcionamiento es el de docencia, entre las $9 \mathrm{y}$ las $14 \mathrm{~h}$ y entre las 16 y 20 h. En período "no lectivo" (entre el 1 de julio y 31 de agosto, Navidad y Semana Santa) el horario de apertura es de $9 \mathrm{~h}$ a $14 \mathrm{~h}$ y de $16 \mathrm{~h}$ a $21 \mathrm{~h}$, abriendo sólo por las mañanas durante la primera quincena de agosto (Universidad de Santiago de Compostela, 2011). El número de usuarios del edificio es de unas 2.200 personas; la mayoría son estudiantes (en torno a 2.100), mientras que los cien restantes son profesores y personal de administración y servicios. Además, existen otros usuarios en un número variable como: personal matriculado en cursos de especialización, tercer ciclo y visitantes.

El edificio posee cinco plantas y un semisótano. Así mismo, el edificio está dividido en tres alas que se denominan Decanato, Conserjería y Aulas; los dos primeros se utilizan para despachos de profesores, seminarios y usos administrativos, mientras que la parte denominada Aulas se utiliza con fines docentes. Además existe un patio interno abierto al exterior, que ilumina con luz natural los pasillos interiores de la zona de aulas de la Planta Baja, este patio se denomina Claustro.

\subsection{DESCRIPCIÓN DE LA INSTALACIÓN ELÉCTRICA Y DE ALUMBRADO}

La Facultad de Derecho dispone de suministro en alta tensión con entrada a 20 $\mathrm{kV}$ proveniente de la red general de la USC en el Campus Sur de Santiago de Compostela; este transformador tiene potencia $250 \mathrm{kVA}$ y transforma la corriente en trifásica a $400 \mathrm{~V} / 230 \mathrm{~V}$. Los equipos eléctricos más importantes son dos ascensores y dos máquinas de aire acondicionado de potencia eléctrica $5 \mathrm{~kW}$; el resto del consumo se encuentra muy disperso entre ordenadores, impresoras, estufas eléctricas, proyecto- 
res,... Respecto a la potencia instalada, no se aprecian problemas importantes de sobrecargas; en el histórico los máximos valores de potencia eléctrica demandada para fuerza han sido de unos $100 \mathrm{kVA}$. En la distribución interna la única posibilidad de salto de térmicos es en la Planta Tercera por la existencia de estufas eléctricas (esta planta tiene una temperatura ambiente más baja). La compra de energía eléctrica se realiza en seis períodos distintos, con un precio que en el año 2007 iba desde los $6 \mathrm{c} €$ por $\mathrm{kWh}$ (el período nocturno, el más barato) a los $14 \mathrm{c} € / \mathrm{kWh}$ por las mañanas de invierno (el más caro); haciendo una media ponderada para todo el Campus Sur de la Universidad de Santiago encontramos que el precio de cada $\mathrm{kWh}$ en ese momento eran $11 \mathrm{c} € / \mathrm{kWh}$.

En cuanto al consumo eléctrico mensual, este está en torno a los $15.000 \mathrm{kWh}$ mensuales (Figura 3), siendo los meses con mayor consumo: enero, marzo, octubre y noviembre; el mes con menor consumo es evidentemente agosto. El consumo anual total es del orden de unos $180.000 \mathrm{kWh}$ cada año; si se calcula el consumo por cada $\mathrm{m}^{2}$ tenemos que este es de unos $11 \mathrm{kWh} / \mathrm{m}^{2}$ y año. A la hora de valorar este ratio de consumo se puede afirmar que está en los parámetros de edificios similares.

Figura 3. Consumo medio de energía eléctrica activa (en kWh) en la Facultad de Derecho. Período 2005-2008.

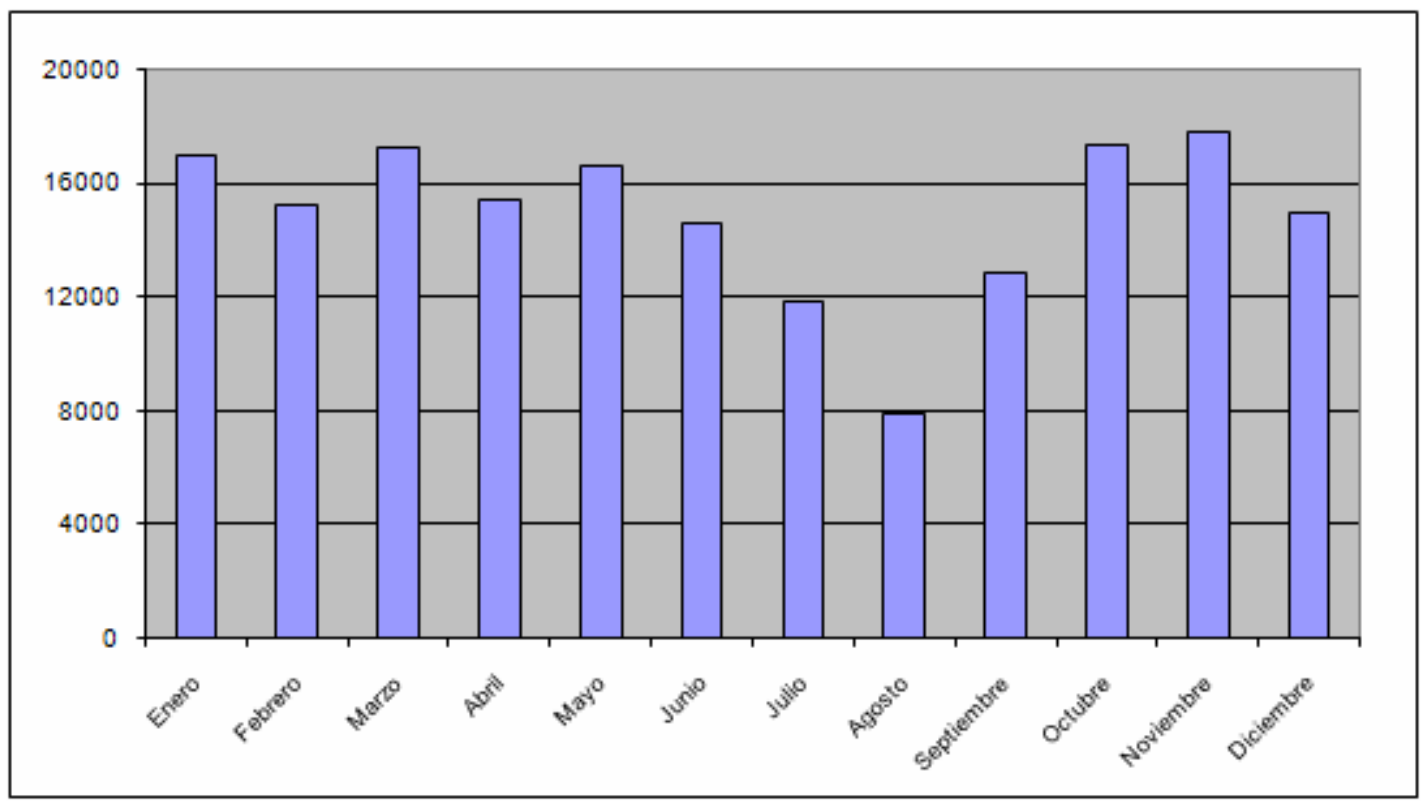

Fuente: Elaboración propia. 
En cuanto a la instalación de alumbrado debemos decir que ésta es muy variada aunque abundan los equipos fluorescentes en los pasillos y vestíbulos (la tipología más común son grupos de $4 \times 36 \mathrm{~W}$ ó $4 \times 18 \mathrm{~W}$ en pasillos), en los despachos y aulas suelen usarse también fluorescentes $\mathrm{y}$, en menor medida, bombillas de bajo consumo. Existen unos 3.000 tubos fluorescentes de $36 \mathrm{~W}, 700$ tubos fluorescentes de $18 \mathrm{~W}$ y unas cien bombillas de bajo consumo; siendo la potencia actual instalada de unos 130 $\mathrm{kW}$. Los equipos fluorescentes están dotados de reactancias electromagnéticas; no obstante, el que el factor de potencia sea bajo no es importante porque en el punto de entronque del Campus Sur existe un sistema de corrección automático (el cual corrige el factor de potencia de toda la red de distribución de la Universidad). La capacidad máxima para alumbrado son unos $200 \mathrm{kVA}$, lo que supone que la carga superior a la de fuerza. Además, de la instalación eléctrica de la Facultad también depende una parte del alumbrado exterior, detectándose en éste que hay una zona con baja radiación.

En 2006 se realizó una auditoría energética de la Facultad de Derecho de la USC en la que se detectaron diferentes problemas, siendo los más graves la elevada transmisión de calor a través de cerramientos deteriorados. Otros problemas de menor entidad fueron: las deficiencias de la instalación eléctrica (resueltos entre 2007 y 2009), las temperaturas excesivas en las plantas sótano y primero, la falta de renovación de aire en la Escuela de Práctica Jurídica (Planta Sótano), las rejillas tipo Graven en el Claustro y la avería de los mecanismos Telesco de cierre automático de las puertas principales (que permanecían demasiado tiempo abiertas).

En cuanto a las deficiencias más importantes en la instalación eléctrica se presentaban una baja intensidad lumínica en la Sala de Lectura, automatización del alumbrado en la zona del Claustro, automatización de alumbrado de vestíbulos de Plantas Segunda y Tercera y problemas de seguridad.

La resolución de las deficiencias se llevo a cabo en varias etapas. En 2007 se realizó la mejora del alumbrado de la Sala de Lectura dejando en espera aquellas actuaciones con las que se pudieran conseguir ayudas a fondo perdido (subvenciones) para la mejora de la eficiencia energética del edificio. Aprovechando la convocatoria anual de ayudas de 2008 del Instituto Enerxético de Galicia (INEGA, 2011) se implementó la automatización del Claustro.

En el año 2008 se firmó un Convenio con la Consellería de Medio Ambiente (Gobierno Autonómico) para efectuar mejoras que consiguiesen la reducción de emisiones de $\mathrm{CO}_{2}$ incluyéndose en esta actuación la mejora de los cerramientos exteriores y el aislamiento de tuberías en plantas Baja y Sótano. Entre las medidas en instalaciones eléctricas se acometió el alumbrado inteligente en los vestíbulos de las Plantas Segunda y Tercera. 


\section{RESULTADOS: ACTUACIONES PARA LA MEJORA DEL} ALUMBRADO EN LA FACULTAD

\subsection{MEJORA DEL ALUMBRADO EN SALA DE LECTURA}

La primera actuación realizada después de la auditoría fue en la Sala de Lectura (Figura 4); esta sala tiene dos zonas diferentes denominadas Fachada Sur y Zona General; la primera tenía un alumbrado en buenas condiciones que se había renovado en 2002 mientras que la Zona General contaba con una instalación deteriorada; la Zona General tiene una superficie de $260 \mathrm{~m} 2$ y contaba con una distribución no simétrica de ochenta y cuatro pantallas de 4x36 W (potencia total $12.096 \mathrm{~W}$ ), que además estaban muy deterioradas. En el año 2006 se realizaron medidas lumínicas en la Zona General a las 12 de la mañana en veinticuatro puntos a una altura de trabajo de $1 \mathrm{~m}$; como resultado de estas medidas se detectó un valor medio de 298 lux y un valor mínimo de 145 lux; el factor de distribución mínimo/media era de 0,46. Podemos apreciar en la Tabla 1 las medidas obtenidas en 2006.

Figura 4. Puntos de medida de intensidad lumínica en la Sala de Lectura de la Planta Baja.

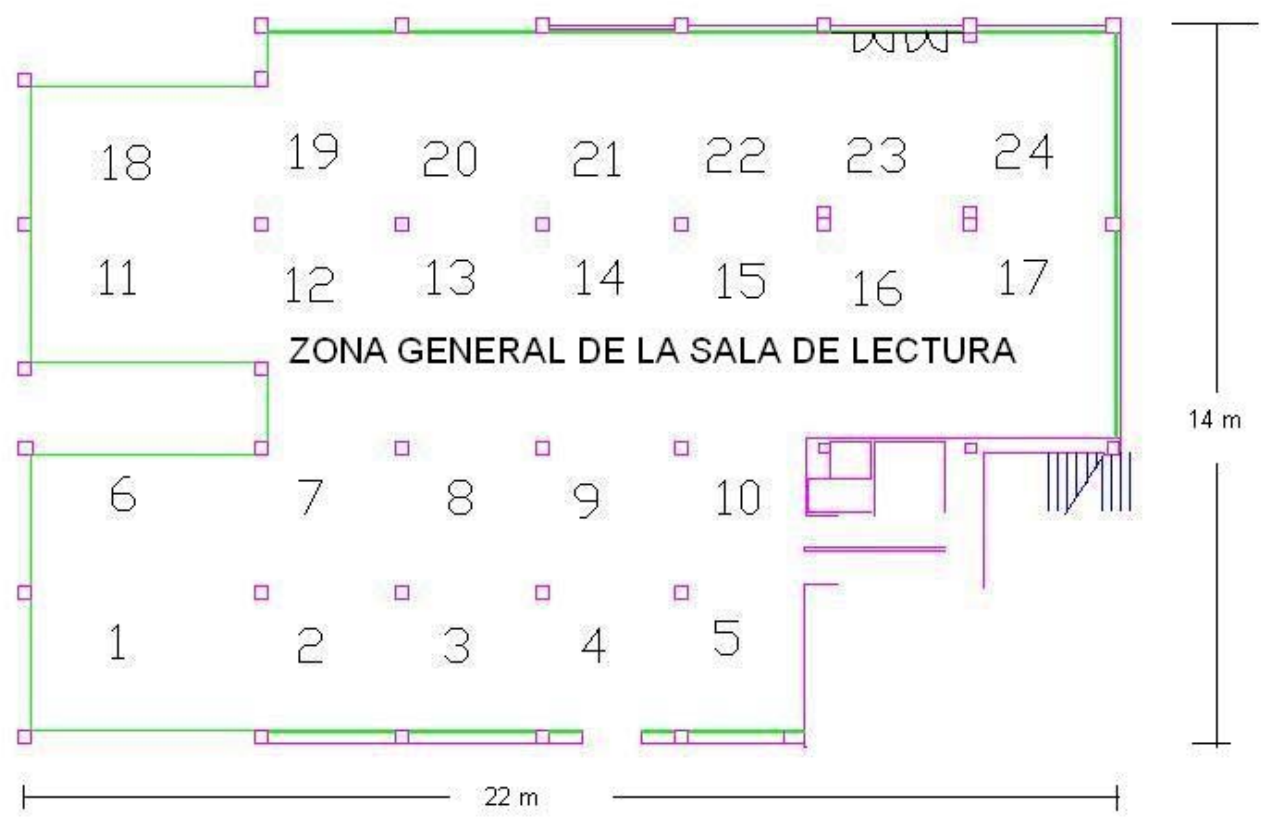

Fuente: Elaboración propia. 
Tabla 1. Medidas obtenidas de intensidad lumínica en 2006.

\begin{tabular}{|c|c|c|c|}
\hline Punto de Medida & Iluminación (lux.) & Punto de Medida & Iluminación (lux.) \\
\hline 1 & 530 & 13 & 360 \\
\hline 2 & 230 & 14 & 310 \\
\hline 3 & 190 & 15 & 285 \\
\hline 4 & 160 & 16 & 280 \\
\hline 5 & 145 & 17 & 280 \\
\hline 6 & 440 & 18 & 445 \\
\hline 7 & 290 & 19 & 315 \\
\hline 8 & 300 & 20 & 190 \\
\hline 9 & 265 & 21 & 350 \\
\hline 10 & 270 & 22 & 300 \\
\hline 11 & 460 & 23 & 340 \\
\hline 12 & 380 & 24 & 200 \\
\hline \multicolumn{2}{|c|}{ Media: } & \multicolumn{2}{|c|}{289 lux } \\
\hline \multicolumn{2}{|c|}{ Máxima: } & \multicolumn{2}{|c|}{530 lux } \\
\hline \multicolumn{2}{|c|}{ Mínima: } & \multicolumn{2}{|c|}{145 lux } \\
\hline \multicolumn{2}{|c|}{ Factor uniformidad (mín/media) } & \multicolumn{2}{|c|}{$\mathbf{0 , 5 0}$} \\
\hline
\end{tabular}

Fuente: Elaboración propia.

En 2007 se renovó completamente el alumbrado de la Zona General de la Sala de Lectura con una nueva instalación de noventa y cinco pantallas de $4 \times 18 \mathrm{~W}$, con una distribución más homogénea (aumentó el número de pantallas pero se rebajó la potencia unitaria de cada una de ellas) y la nueva potencia es de $6.840 \mathrm{~W}$; pese a una reducción de la potencia de un $43 \%$ encontramos que realizadas las medidas experimentales el nuevo valor medio es de 491 lux, el máximo son 680 luxes y el mínimo 390 lux mientras que el nuevo factor de uniformidad es 0,79 . El coste total de la actuación fueron $10.000 €$ (Impuestos incluidos).

Con esta nueva distribución hemos ahorrado $16.800 \mathrm{kWh} /$ año (se ha reducido la potencia $5,1 \mathrm{~kW}$ con un funcionamiento de unas 2.800 horas anuales de funcionamiento) y un ahorro anual de unos $1.850 €$ en energía eléctrica, a lo que le deberemos 
sumar unos $150 €$ por gastos de mantenimiento no efectuado (I.V.A. incluido); el periodo de retorno fue de cinco años ${ }^{1}$, y la evolución de los consumos ha sido la esperada. Es importante citar que debido a que el periodo de retorno es razonablemente corto no se considera que la evolución de precios de la electricidad y costes de mantenimiento sean superiores al IPC aunque es de esperar que esto se cumpla. El periodo se podría reducir a lo sumo en medio año.

El ahorro de $16.800 \mathrm{kWh}$ supone dejar de emitir 2,8 toneladas de $\mathrm{CO}_{2}$ cada año, siendo el coeficiente de emisiones de $0,166 \mathrm{~kg} \mathrm{CO}_{2} / \mathrm{kWh}$ utilizando metodología del Instituto de Diversificación y Ahorro Energético para 2007 (CENEAM, 2011). Además debemos citar que han desaparecido las quejas por parte de los usuarios de baja intensidad lumínica, ya que la distribución actual de luminarias es mucho más eficiente. En la Tabla 2 podemos ver los resultados obtenidos mediante esta actuación.

Tabla 2. Principales datos de la actuación en la Sala de Lectura.

\begin{tabular}{|c|c|c|c|}
\cline { 2 - 3 } \multicolumn{1}{c|}{} & $\begin{array}{c}\text { Potencia Total } \\
\text { Instalada }\end{array}$ & Iluminación media & $\begin{array}{c}\text { Ahorro con la Actua- } \\
\text { ción }\end{array}$ \\
\hline Año 2007: & $12.096 \mathrm{~W}$ & 298 lux. & $\begin{array}{c}16.800 \mathrm{kWh} / \text { año } \\
2000 € / \text { año }\end{array}$ \\
\hline Año 2008: & $6840 \mathrm{~W}$ & $491 \mathrm{lux}$. & \\
\hline
\end{tabular}

\subsection{INSTALACIÓN DE UN SISTEMA DE ALUMBRADO INTELIGENTE EN CLAUSTRO}

La Auditoría detectó una deficiencia importante en cuanto al excesivo alumbrado natural en los pasillos y vestíbulos; durante su realización se llevaron a cabo múltiples visitas al edificio en las que se observó que a plena luz del día había muchas luminarias encendidas, incluso con abundancia de luz solar. Consultado el personal de la Conserjería nos informan que se debe a que los interruptores están accesibles a todos los usuarios, que encienden continuamente las luces y el apagado es ocasional. Para solucionar esto se decidieron realizar dos instalaciones de alumbrado automatizado; uno de estos alumbrados sobre la zona del Claustro y Planta Primera de Aulas y el otro en las plantas Segunda y Tercera del Edificio-Zona de Conserjería. Nos referimos a un alumbrado inteligente como el control de las luminarias en función de un parámetro ajeno; en este caso el parámetro es la radiación solar y está medido mediante una fotocélula que enciende/apaga las luces cuando no se alcanza un límite de radiación solar previamente establecido (actualmente $50 \mathrm{~W} / \mathrm{m} 2$ ). Estas actuación se realizó en 2008 .

${ }^{1}$ Debido a que el periodo de retorno es razonablemente corto no se considera que la evolución de precios de la electricidad y costes de mantenimiento sean superiores al IPC aunque es de esperar que esto se cumpla. El periodo se podría reducir a lo sumo en medio año. 
En la Figura 5 se puede apreciar un croquis de dicha actuación para la Planta Baja.

Figura 5. Croquis con reseña de la actuación llevada a cabo.

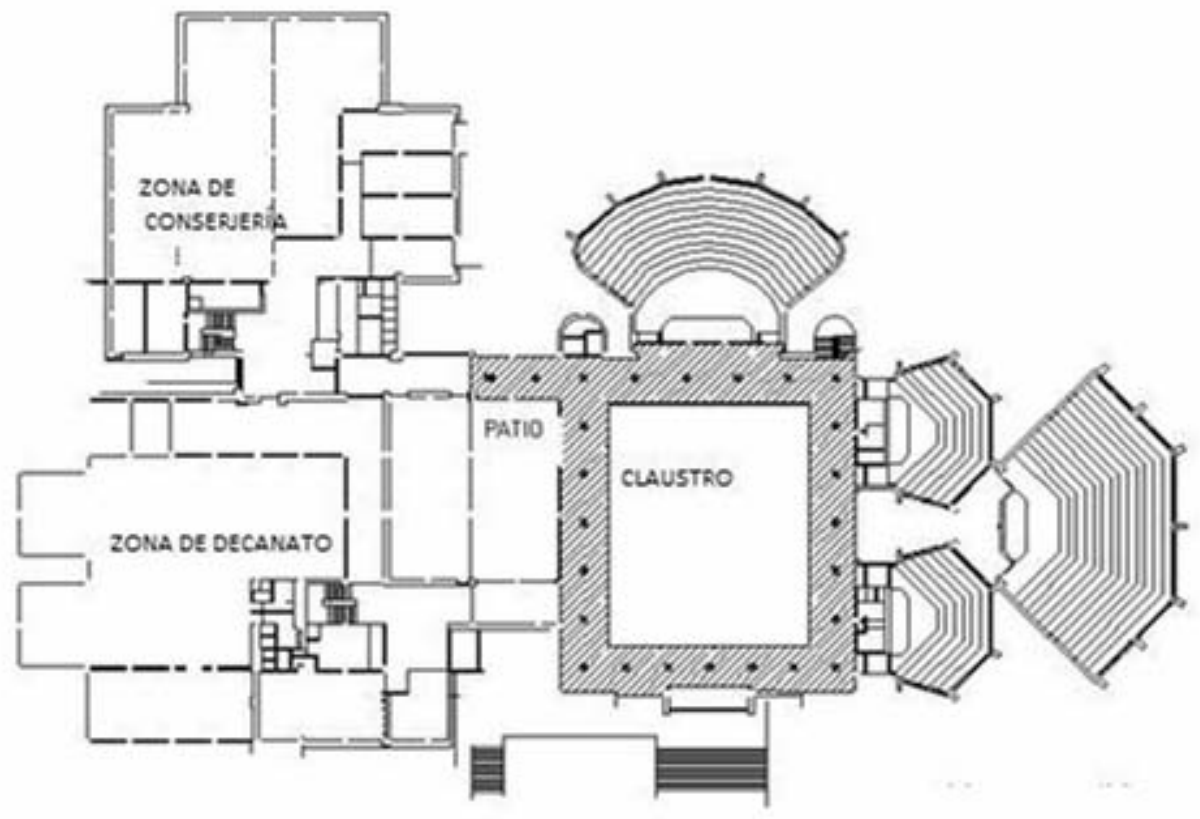

Fuente: Elaboración propia.

La automatización del alumbrado abarcaba las zonas del Claustro y Planta Primera. La nueva instalación está formada por veinticinco pantallas de 4x $36 \mathrm{~W}$ (potencia total $3.600 \mathrm{~W}$ ) en la Planta Baja y ocho pantallas de 4x36 W en la Planta Primera, que originariamente se encendían y apagaban mediante interruptores accesibles al público; el nuevo alumbrado mantiene la potencia instalada $(4,8 \mathrm{~kW})$ y reduce el funcionamiento durante unas 600 horas al año, por lo que además del consumo también había un deterioro importante por mantenimiento. La nueva instalación centraliza el control de todas las luminarias en una fotocélula, ahorrando $2.880 \mathrm{kWh} /$ año y evitando la emisión de $480 \mathrm{~kg}$ de $\mathrm{CO}_{2}$ por año. Debido a que el precio de cada $\mathrm{kWh}$ en 2008 era de unos 11 céntimos de euro por cada kWh, el ahorro anual es de unos $316 €$ el primer año (Tabla 3), a lo que se le añaden $80 €$ por cambio de luminarias que deja de hacerse cada año (mediante el nuevo alumbrado se tienen que realizar menos tareas de mantenimiento porque las luminarias tardan más en gastarse además de cambiarse de forma simultánea). El precio de la actuación ha sido de $10.000 €$ (impuestos incluidos), contando con una subvención de un 22\% a cargo del Instituto Enerxético de Galicia, por lo que el precio real fueron unos $7.800 €$. Para el cálculo del periodo de retorno debemos considerar que la evolución del precio de la electricidad y de los 
gastos de mantenimiento evolucionarán por encima del Índice de Precios al Consumo (I.P.C.), por lo que debemos hacer un cálculo de precios actualizados ya que en un periodo tan largo esta diferencia debe ser considerado; en nuestro caso hemos considerado que los costes de energía eléctrica y mantenimiento aumentan un $1 \%$ por encima del IPC, como se muestra en la Tabla 3. En esta tabla se puede ver que el periodo de retorno con subvención son 17 años y sin ayudas se alarga hasta los 21,5 años.

Tabla 3. Cálculo del periodo de retorno del alumbrado inteligente para la zona de Claustro.

\begin{tabular}{|c|c|c|c|c|c|}
\hline Año & $\begin{array}{c}\text { Precio electricidad } \\
(€ / \mathbf{k W h}) \\
\end{array}$ & $\begin{array}{c}\text { Ahorro electricidad } \\
(€)\end{array}$ & $\begin{array}{c}\text { Mantenimiento } \\
(€) \\
\end{array}$ & $\begin{array}{c}\text { Coste total año } \\
(€)\end{array}$ & $\begin{array}{c}\text { Total acumulado } \\
(€) \\
\end{array}$ \\
\hline 0 & 0,110 & 316,8 & 80,0 & 396,8 & 396,8 \\
\hline 1 & 0,111 & 320,0 & 80,8 & 400,8 & 797,6 \\
\hline 2 & 0,112 & 323,2 & 81,6 & 404,8 & 1202,3 \\
\hline 3 & 0,113 & 326,4 & 82,4 & 408,8 & 1611,2 \\
\hline 4 & 0,114 & 329,7 & 83,2 & 412,9 & 2024,1 \\
\hline 5 & 0,116 & 333,0 & 84,1 & 417,0 & 2441,1 \\
\hline 6 & 0,117 & 336,3 & 84,9 & 421,2 & 2862,3 \\
\hline 7 & 0,118 & 339,7 & 85,8 & 425,4 & 3287,8 \\
\hline 8 & 0,119 & 343,0 & 86,6 & 429,7 & 3717,4 \\
\hline 9 & 0,120 & 346,5 & 87,5 & 434,0 & 4151,4 \\
\hline 10 & 0,122 & 349,9 & 88,4 & 438,3 & 4589,7 \\
\hline 11 & 0,123 & 353,4 & 89,3 & 442,7 & 5032,4 \\
\hline 12 & 0,124 & 357,0 & 90,1 & 447,1 & 5479,5 \\
\hline 13 & 0,125 & 360,5 & 91,0 & 451,6 & 5931,1 \\
\hline 14 & 0,126 & 364,2 & 92,0 & 456,1 & 6387,2 \\
\hline 15 & 0,128 & 367,8 & 92,9 & 460,7 & 6847,9 \\
\hline 16 & 0,129 & 371,5 & 93,8 & 465,3 & 7313,2 \\
\hline 17 & 0,130 & 375,2 & 94,7 & 469,9 & 7783,1 \\
\hline 18 & 0,132 & 378,9 & 95,7 & 474,6 & 8257,8 \\
\hline 19 & 0,133 & 382,7 & 96,6 & 479,4 & 8737,1 \\
\hline 20 & 0,134 & 386,6 & 97,6 & 484,2 & 9221,3 \\
\hline 21 & 0,136 & 390,4 & 98,6 & 489,0 & 9710,3 \\
\hline 22 & 0,137 & 394,3 & 99,6 & 493,9 & 10204,2 \\
\hline
\end{tabular}

Fuente: Elaboración propia. 
En la Figura 6 se muestra una fotografía (figura de la izquierda) de la actuación implementada en el Claustro de la Facultad de Derecho.

Figura 6. Vista del alumbrado del Claustro (Izquierda) y Vestíbulo (Derecha) del Edificio de la Facultad de Derecho de la USC.
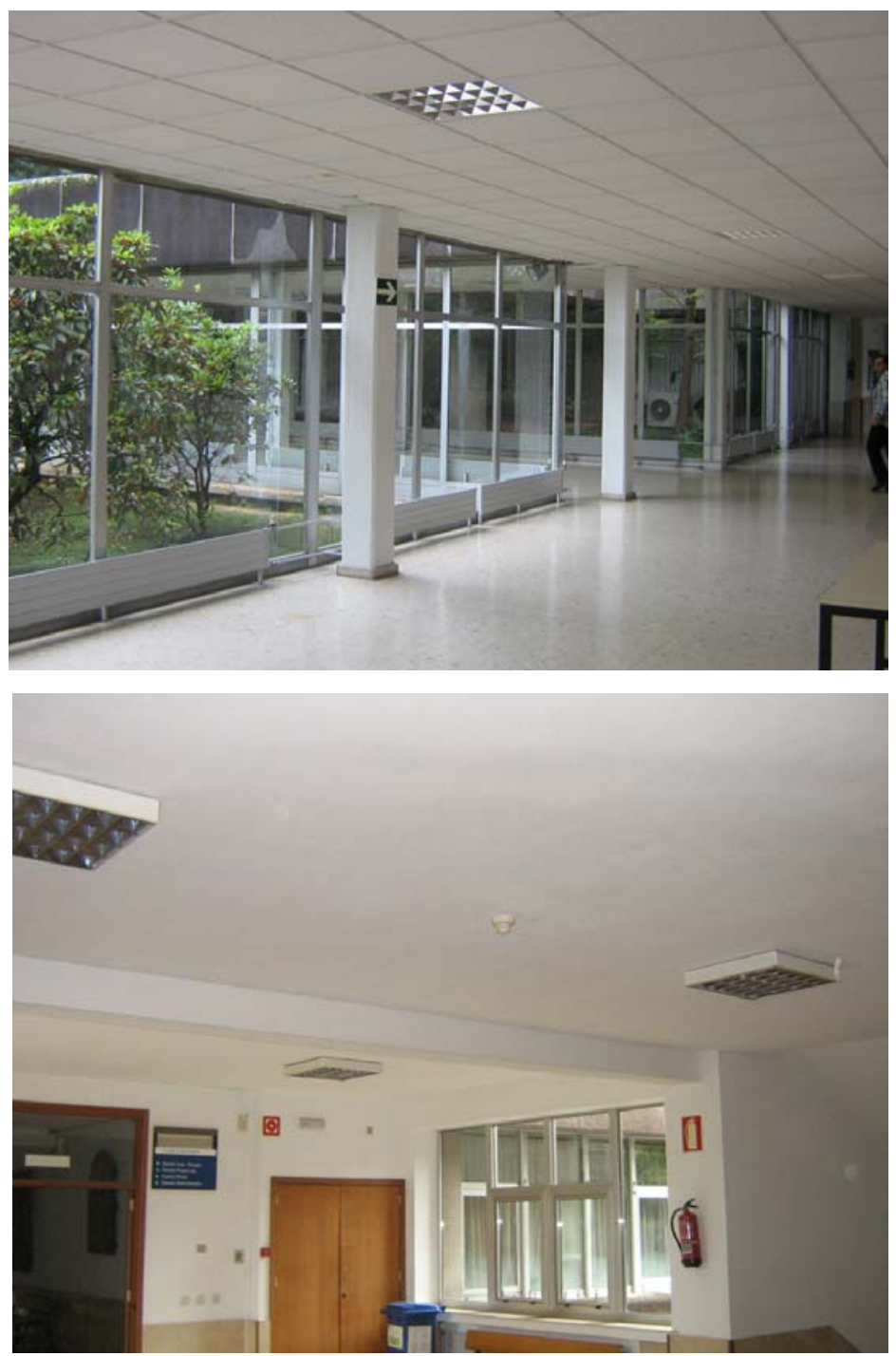

Fuente: Elaboración propia. 


\subsection{INSTALACIÓN DE CÉLULAS FOTOELÉCTRICAS EN VESTÍBULOS (PLANTAS SEGUNDA Y TERCERA)}

La tercera y última actuación se llevó a cabo en 2009; consistió en optimizar el alumbrado natural de las plantas Segunda y Tercera de la Zona de Aulas, en la zona del Vestíbulo (Figura 6 - Derecha). En estas zonas existe luz natural y los usuarios podían realizar el encendido y apagado manual de las luminarias, repitiéndose a menudo la situación de luminarias encendidas con abundante luz solar. En estas plantas se ha colocado una célula fotoeléctrica que regula el apagado y encendido según la radiación solar; esta célula regula el encendido de ocho pantallas de 4x36 W (cuatro en cada una de las plantas), por lo que la potencia regulada son $1.152 \mathrm{~W}$. Se recorta aproximadamente el funcionamiento de dichas luminarias durante 500 horas al año, lo que supone un ahorro de $550 \mathrm{kWh} /$ año y unos $92 \mathrm{~kg}$ de $\mathrm{CO}_{2}$ cada año. El coste ahorrado es de $65 € /$ año a lo que se deben añadir $15 €$ que se dejan de gastar por mantenimiento y por lo tanto el ahorro anual son $80 € /$ año. El precio de esta actuación ha sido de 1.000 euros (I.V.A. incluido), contando, a posteriori, con una subvención del 50\% a cargo de la Consellería de Medio Ambiente de la Xunta de Galicia. Considerando que el precio de la energía eléctrica y de costes de mantenimiento son iguales al IPC los períodos de retorno serían de 12,5 años y 6,25 años, en el caso de consideremos que los costes de mantenimiento y electricidad superen al Índice de Precios al Consumo (I.P.C.) en un $1 \%$ estos períodos se acortarían a doce y seis años, respectivamente.

\section{DISCUSIÓN: BALANCE ENERGÉTICO Y ECONÓMICO}

Con el fin de visualizar el ahorro energético y las repercusiones económicas de las diferentes actuaciones llevadas a cabo en el edificio se muestra en la Tabla 4 un resumen.

Tabla 4. Ahorro energético y cálculo del periodo de retorno de las inversiones realizadas para la mejora de la Facultad de Derecho.

\begin{tabular}{|c|c|c|c|c|}
\hline \multicolumn{2}{|c|}{ mejora de la Facultad de Derecho. } & $\begin{array}{l}\text { Sala de } \\
\text { lectura }\end{array}$ & $\begin{array}{c}\text { Alumbrado } \\
\text { claustro }\end{array}$ & $\begin{array}{c}\text { Alumbrado } \\
\text { plantas } 2^{\mathrm{a}} \text { y } 3^{\mathrm{a}}\end{array}$ \\
\hline & - Año de entrada en vigor: & 2007 & 2008 & 2009 \\
\hline \multirow{4}{*}{$\begin{array}{l}0 \\
\frac{\pi}{\pi} \\
\frac{\pi}{0} \\
\frac{0}{\&}\end{array}$} & - Ahorro en electricidad (kWh/año): & 16800 & 2880 & 550 \\
\hline & - Ahorro eléctrico primer año $(€)$ : & 1850 & 320 & 65 \\
\hline & - Ahorro mantenimiento primer año $(€)$ : & 150 & 80 & 15 \\
\hline & - Ahorro total anual (€): & 2000 & 400 & 80 \\
\hline & - Coste sin subvención ( $€$, I.V.A. incluido): & 10000 & 10000 & 1000 \\
\hline & - Coste con subvención ( $€$, I.V.A. incluido): & ---- & 7800 & 500 \\
\hline & - Periodo de retorno sin subvención: & 5 años & 21,5 años & 12 años \\
\hline & - Periodo de retorno con subvención: & ---- & 17 años & 6 años \\
\hline
\end{tabular}

Fuente: Elaboración propia. 


\section{CONCLUSIONES}

En el presente artículo se han mostrado las actuaciones llevadas a cabo con el objetivo de sanear la instalación de alumbrado del Edificio. Se trata de un edificio de la década de los setenta sobre el que se realizaron tres actuaciones con el fin de ahorrar energía eléctrica $(20.320 \mathrm{KWh} / \mathrm{año})$, mediante dos alumbrados regulados por la intensidad de luz exterior y la sustitución del alumbrado completo de la Sala de Lectura. Como consecuencia de las actuaciones implementadas se han reducido las emisiones de $\mathrm{CO}_{2}$ del Edificio objeto de estudio en 3,4 toneladas.

\section{AGRADECIMIENTOS}

Al Instituto Energético de Galicia (IN.E.Ga) y a la Consellería de Medioambiente de la Xunta de Galicia por la financiación parcial de este trabajo y de las actuaciones llevadas a cabo en el Edificio.

\section{REFERENCIAS BIBLIOGRÁFICAS}

BALLENILLA, Fernando (2004). El final del petróleo barato, El Ecologista, No 40, 20-23.

RUIZ BEVIÁ, Francisco (1981). Crisis energética y materias primas en la industria química, Universidad de Alicante:

http://web.ua.es/en/protocolo/documentos/lessons/lesson-opening-81-82.pdf [Consulta 17 de agosto de 2012]

BRITISH PETROLEUM (2011). B.P. Statistical Review of Energy, Londres. Disponible en:

http://www.bp.com/assets/bp_internet/globalbp/globalbp_uk_english/reports_and publica-

tions/statistical_energy_review_2011/STAGING/local_assets/pdf/statistical_revie w_of_world_energy_full_report_2011.pdf [Consulta 20 de agosto de 2012]

AMESTOY ALONSO, José (2000). Consideraciones en torno al impacto medioambiental de las fuentes de energía, Lurralde: Investigación y espacio, $\mathrm{N}^{\mathrm{o}} 23,75-91$.

BUSTELO, Pablo (2008). Seguridad energética con alta dependencia externa: las estrategias de Japón y Corea del Sur, Real Instituto Elcano, Documento de Trabajo $\mathrm{N}^{\mathrm{o}} 16 / 2008$, Madrid.

CAMPOS HERNÁNDEZ, José Manuel (2008). El Protocolo de Kyoto y los Gases de Efecto Invernadero: Vigilancia o catastrofismo, Ingeniería y territorio, $\mathrm{N}^{\circ} 82$, , 3037.

FERNÁNDEZ LÓPEZ, MARÍA ÁNGELES; FERNÁNDEZ FERNÁNDEZ, YOLANDA; OLMEDILLAS BLANCO, BLANCA (2008). Los derechos de emisión como instrumento para alcanzar Kyoto, Revista de Estudios Empresariales, Segunda Época, No 2, 93-117. 
DELGADO-IRIBARREN GARCÍA-CAMPERO, Manuel (1984). El Plan Energético Nacional (P.E.N.) 1983 en: Revista de Administración Pública, No 104, 449-462.

SÁNCHEZ TEMBLEQUE, Luis Jesús (2011). La estrategia de ahorro y eficiencia energética en España 2004-2012 en: Cuadernos de sostenibilidad y patrimonio natural, $N^{\circ} 4$, (2004), 86-99. B.P. Statistical Review of Energy, Londres.

FORO NUCLEAR (2011). Prontuario Energía 2011, Madrid, 2011: Disponible en: http://www.foronuclear.org/images/stories/recursos/publicaciones/2011/ENERGA 2011.pdf [Consultado a 15 de junio de 2012].

UNIVERSIDAD DE SANTIAGO DE COMPOSTELA (2011). Guía de la Facultad de Derecho. Disponible en: http://www.usc.es/fac_dereito/index.htm [Consultado a 2 de julio de 2012].

WEB OFICIAL DEL INSTITUTO ENERGÉTICO DE GALICIA (INEGA) (2011). http://www.inega.es [Consultado a 3 de julio de 2012].

CENTRO NACIONAL DE EDUCACION AMBIENTAL - CENEAM (2011). Emisiones de $\mathrm{CO}_{2}$ del CENEAM, Madrid, 2011. Disponible en: http://www.marm.es/es/ceneam/recursos/mini-portales-tematicos/emisiones_tcm712904.pdf [Consultado a 10 de julio de 2012]. Pág. 10. 